\title{
Delegation von Aufgaben im Management
}

\author{
Relevanz und Umsetzung
}

Die Zunahme zeitlicher Engpässe, z. B. durch die Verschlankung von Managementstrukturen, lässt die zielgerichtete Delegation von Aufgaben zu einer Kernkompetenz des Managements werden. Es zeigt sich allerdings, dass effektives und effizientes Delegieren eine große Herausforderung darstellt. Dabei auftretende Probleme werden im Rahmen dieses Beitrags thematisiert. Es wird ein Vorgehensschema präsentiert, mit welchem den Problemen begegnet werden kann.

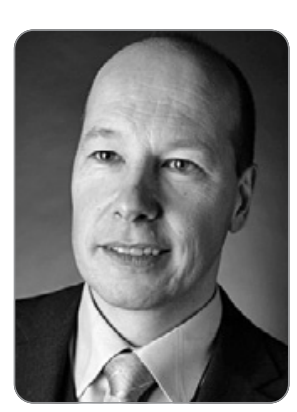

Prof. Dr. Heinz Ahn

ist Leiter des Instituts für Controlling und Unternehmensrechnung der Technischen Universität Braunschweig. Seine bevorzugten Themengebiete sind Kostenrechnung und Kostenmanagement, effektivitäts- und effizienzorientierte Planungskonzepte sowie Leistungssteuerung. Er ist Akademischer Leiter des Technologietransferzentrums "Center for Performance Management" der Innovationsgesellschaft der TU Braunschweig $\mathrm{mbH}$.

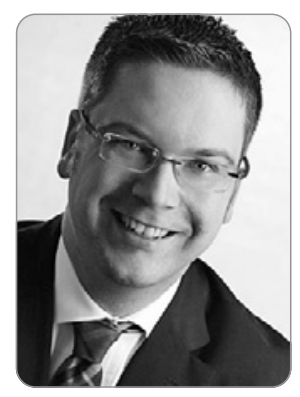

\section{Prof. Dr. Marcel Clermont}

ist Inhaber der Professur für Betriebswirtschaftslehre mit Schwerpunkt Rechnungswesen und Controlling an der Dualen Hochschule Gera-Eisenach. Er hat vielfältige Beratertätigkeiten wahrgenommen und ist Mitgesellschafter der StraTax Consulting GbR. Er forscht und lehrt auf dem Gebiet der internen Unternehmensrechnung und im Speziellen im Bereich des Performance Managements.

Stichwörter: Delegation, Management by Delegation, Vorgehensschema, Tätigkeitsklassifikation, Verantwortungsübertragung

1. Delegation als notwendige Maßnahme des Zeitmanagements

Eine der zentralen Herausforderungen an Führungskräfte ist es, die Balance zwischen der Ausübung operativer Routinetätigkeiten und strategisch relevanter Managementtätigkeiten zu bewahren. Letztere stellen den eigentlichen
Aufgabenkern des Managements dar und können abstrakt in Tätigkeiten der Planung, der Kontrolle und des Lernens unterteilt werden (vgl. Ahn, 2003, S. 71 ff.). Konkret geht es z. B. um die Kosten-Nutzen-Abschätzung moderner Data Analytics Software, die Einarbeitung in neue Ansätze des Benchmarkings oder das Überdenken der Prozessabläufe in einer bestimmten Abteilung.

Solche Managementtätigkeiten kommen in der Praxis allerdings häufig zu kurz. Die möglichen Ursachen dafür sind vielfältig: Sie reichen von der wachsenden Informationsmenge über eine zunehmende Dichte zu beachtender Regularien hin zur Umsetzung von Konzepten des Lean Management mit der Konsequenz sich vergrößernder Verantwortungsbereiche. Sofern betroffene Führungskräfte selbst keinen Einfluss auf derartige Entwicklungen nehmen können, ist es für sie essentiell, sich anderweitig Freiraum d. h. Zeit - für strategische Tätigkeiten zu verschaffen. Ansonsten droht die Gefahr, dass Routinetätigkeiten nach und nach die gesamte Arbeitszeit beanspruchen.

Eine naheliegende Maßnahme ist die Delegation von Routinetätigkeiten an ausführende MitarbeiterInnen. Ihre Umsetzung stößt im Arbeitsalltag freilich auf zahlreiche Hürden. Diese werden im Folgenden insoweit thematisiert, wie sie der Gestaltungshoheit einer delegierenden Person unterliegen. Ziel ist es, mit Blick auf eine erfolgreiche Delegation wissenswerte Aspekte und hilfreiche Methoden zusammenfassend vorzustellen. Dabei fließen die Einsichten aus einem Coaching-Projekt ein, welches den Impuls für den vorliegenden Beitrag gegeben hat. 


\section{Ein idealtypischer Weg zur erfolgreichen Delegation}

Zum Thema Delegation existiert bereits eine Fülle meist populärwissenschaftlicher Literatur. Eine Synopse der in diesen Ratgebern aufgezeigten Hinweise führt $\mathrm{zu}$ dem in Abb. 1 dargestellten Vorgehensschema, das die einzelnen Schritte eines idealtypischen Delegationsprozesses wiedergibt. Strukturiert in drei Phasen werden diese Schritte in den nachfolgenden Abschnitten skizziert.

\subsection{Problemerkennung}

Delegation ist kein Selbstzweck. Vielmehr ermöglicht sie Führungskräften, Zeit für die Auseinandersetzung mit strategisch relevanten Projekten zu gewinnen. Daneben kann
Delegation eine motivierende Wirkung auf MitarbeiterInnen haben (vgl. z. B. Laux, 1979, S. 20). Obwohl diese Potenziale altbekannt sind, ist ihre durchdachte Nutzung wohl eher die Ausnahme. So verwundert es nicht, dass vor allem im unteren und mittleren Management operative Tätigkeiten (zu sehr) im Fokus stehen. Zu den entsprechenden Warnsignalen gehören:

- länger werdende Arbeitszeiten,

- das Gefühl von Unverzichtbarkeit und

- enge Grenzen des Engagements der MitarbeiterInnen.

Dies können Folgen von Faktoren sein, welche in der Person der Führungskraft selbst begründet liegen. Die folgende Tab. 1 listet schlagwortartig prominente Beispiele solcher Faktoren auf und skizziert ihre Problematik (siehe weiterführend z. B. Boneberg, 2008, S. 169, Gallo, 2012).
Abb. 1: Vorgehensschema für eine erfolgreiche Delegation

Tab. 1: Warum delegieren Sie nicht?

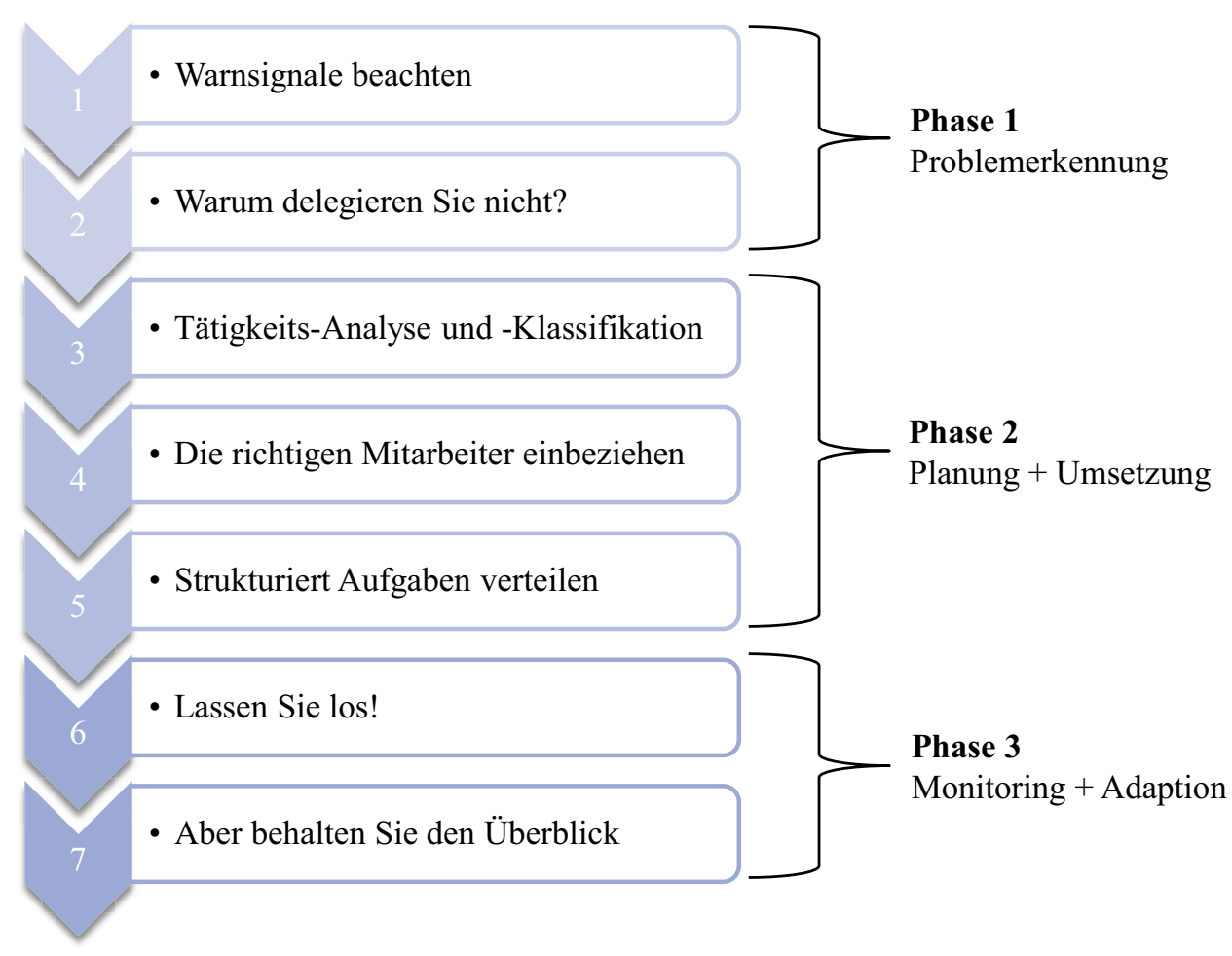

\begin{tabular}{|c|c|}
\hline Schlagwort & Folge: Vernachlässigung zentraler Managementaufgaben \\
\hline $\begin{array}{l}\text { „Es geht schneller, wenn } \\
\text { ich es selber erledige“. }\end{array}$ & \multirow{2}{*}{$\begin{array}{l}\text { Nicht zu delegieren heißt, die Förderung bzw. Forderung der } \\
\text { MitarbeiterInnen zu vernachlässigen. Neuen Aufgaben sind sie } \\
\text { daher immer weniger gewachsen und sie sind zunehmend } \\
\text { frustriert. So entsteht und verstärkt sich schrittweise das Prob- } \\
\text { lem einer unterqualifizierten Belegschaft mit mangelndem } \\
\text { Einsatzwillen. }\end{array}$} \\
\hline $\begin{array}{l}\text { „Meinem Team fehlen } \\
\text { die notwendigen Fähig- } \\
\text { keiten.“ }\end{array}$ & \\
\hline $\begin{array}{l}\text { Priorität } \\
\text { mung als } \\
\text { Experte }\end{array}$ & \multirow{2}{*}{$\begin{array}{l}\text { Als Führungskraft wird man i. d. R. nicht beschäftigt, um sich } \\
\text { auf Detailwissen bzgl. fachlicher Fragen in einem bestimmen } \\
\text { Arbeitsgebiet zu konzentrieren. Vielmehr besteht die Aufgabe } \\
\text { darin, seinen Verantwortungsbereich zukunftsfähig zu gestal- } \\
\text { ten. Besitzt man diese Fähigkeit, ist man - im Gegensatz zum } \\
\text { Fachexperten - auch bereichsübergreifend einsetzbar. }\end{array}$} \\
\hline $\begin{array}{l}\text { Angst vor Bedeutungs- } \\
\text { verlust }\end{array}$ & \\
\hline Hang zu Perfektionismus & $\begin{array}{l}\text { Perfektionismus ist nicht nur ineffizient, er verstärkt auch die } \\
\text { skizzierten Problematiken, indem er Opportunitätskosten sowie } \\
\text { Frust aufgrund mangelnder Akzeptanz verursacht. }\end{array}$ \\
\hline
\end{tabular}




\subsection{Planung und Umsetzung systematischer Delegation}

Es stellt eine Herausforderung dar, Defizite im Delegationsverhalten zu beseitigen, da verinnerlichte Arbeitsroutinen aller Beteiligten aufgebrochen werden müssen. Eine systematische Vorgehensweise kann hier helfen, wobei es naheliegend ist, mit einer Tätigkeitsanalyse und -klassifikation zu beginnen. Die dazu oft propagierte EisenhowerMatrix (vgl. z. B. Knoblauch et al., 2015, S. 27 ff., Seiwert, 2016, S. 115 f.) greift allerdings zu kurz, weil sie darauf fokussiert, unwichtige, aber dringliche Aufgaben zu delegieren. Dies würde auf Dauer nicht nur demotivierend auf die MitarbeiterInnen wirken, sondern insbesondere ihre Potenziale unberücksichtigt lassen.

Deshalb wird ein in Abb. 2 dargestellter, modifizierter Ansatz zur Tätigkeitsklassifikation vorgeschlagen. Neben dem Ausmaß der Entlastung der Führungskraft dient das vorhandene Potenzial der MitarbeiterInnnen zur Aufgabenübernahme als neues Differenzierungskriterium. Dies geht deutlich über den Eisenhower-Ansatz hinaus. Es wird nämlich erforderlich, sich mit den Fähigkeiten der MitarbeiterInnen und ihrer Einsatzbereitschaft auseinanderzusetzen. Dahingehend sollten mit ihnen Gespräche geführt werden, was auch aufkommenden Ängsten bezüglich der geplanten Änderungen der Aufgabenverteilung entgegenwirken kann (vgl. z. B. Laux, 1979, S. 17 ff.).

Auf dieser Basis können nun Management-Tätigkeiten klassifiziert werden. Gemäß Abb. 2 ergeben sich folgende Handlungsempfehlungen:

- Segment A: Wenn Entlastungsausmaß und Mitarbeiterpotenzial als gering eingeschätzt werden, ist keine Delegation empfehlenswert.

- Segment B: Wenn allein das Entlastungsausmaß gering ist, bietet es sich nichtsdestotrotz an zu prüfen, ob durch Delegation andere positive Effekte erzielt werden können, z. B. eine Steigerung des Selbstbewusstseins der

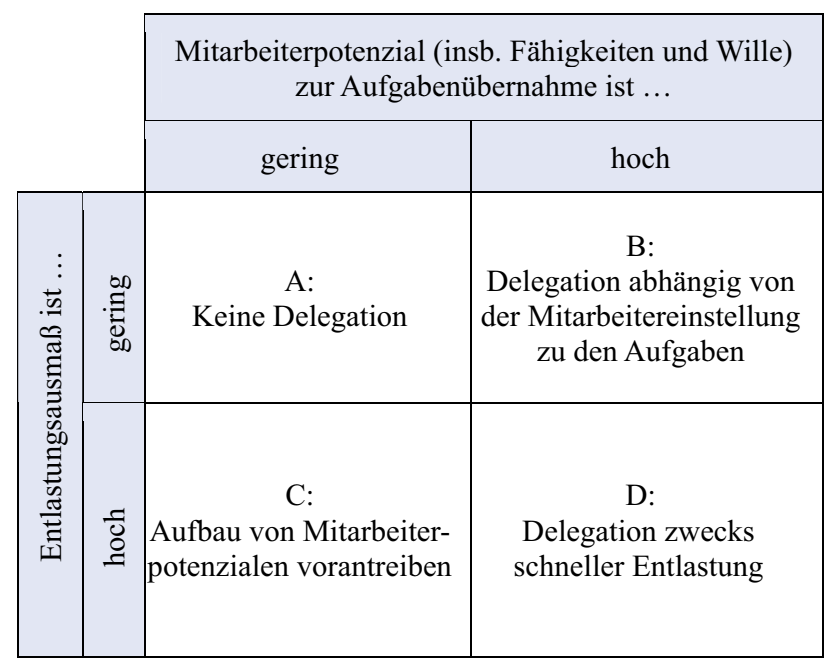

Abb. 2: Tätigkeitsklassifikation unter Einbezug von Mitarbeiterpotenzialen
MitarbeiterInnen.

- Segment C: Wenn allein das Mitarbeiterpotential gering ist, sollte ein systematischer Aufbau entsprechender Potenziale vorangetrieben werden, z. B. durch Schulungen und eine schrittweise Verantwortungsübertragung.

- Segment D: Im Idealfall eines hohen Entlastungsausmaßes und Mitarbeiterpotenzials ermöglicht die Delegation eine schnelle Entlastung der Führungskraft.

Dem Segment C kommt eine besondere Bedeutung zu. Vor allem wenn eine stark überlastete Führungskraft ihre prinzipiell delegierbaren Tätigkeiten gemäß der Matrix klassifiziert, könnte sich herausstellen, dass sich ein Großteil in diesem Segment wiederfinden wird. Dann lässt sich das Problem der Überlastung nicht ad hoc lösen, die Delegationsaufgabe bekommt einen "dynamischen" Charakter. Mehr noch: es tritt offen zutage, dass Versäumnisse hinsichtlich der Mitarbeiterförderung und -forderung vorliegen - damit gewinnt die ohnehin sensible Thematik eine gewisse Brisanz. Darüber sollten sich betroffene Führungskräfte im Klaren sein.

Abschließend sei die oben angesprochene schrittweise Verantwortungsübertragung exemplarisch konkretisiert. Zur Darstellung eignet sich die Beteiligung von MitarbeiterInnen an der Leitung von Projekten, wie sie in Abb. 3 (linke Hälfte) skizziert ist. Vom operativen Monitoring eines Projekts über die Verantwortung seiner Umsetzung und der Auswahl notwendiger Steuerungsmaßnahmen bis hin zur Zielpriorisierung sind die Tätigkeitsinhalte (insbesondere im Sinne eines Übergangs von Aufgaben- zu Verantwortungsdelegation; vgl. Covey 2016, S. 202 ff.) sukzessive ausweitbar. Damit kann eine ebenso sukzessiv angepasste Kontrolle durch die Führungskraft einhergehen (siehe rechte Hälfte von $A b b$. 3).

\subsection{Monitoring und Adaption}

Um sich durch Delegation Freiraum für strategische Tätigkeiten zu schaffen, bedarf es großer Disziplin, denn nur allzu leicht verfällt man in alte Verhaltensmuster. Daher ist das eigene Delegationsverhalten regelmäßig zu reflektieren. Folgende Fragen können dabei helfen:

- Delegiert man tatsächlich wie geplant?

- Vermeidet man Mikromanagement?

- Schafft man es, bei auftretenden Problemen "das Heft nicht sofort wieder selbst in die Hand zu nehmen"?

Das Schlagwort "Lassen Sie los!“ sollte allerdings nicht missinterpretiert werden. Selbstverständlich bedarf es auch Mechanismen, die der Führungskraft einen Überblick über die Aufgabenerfüllung durch die MitarbeiterInnen verschafft. Die Kunst besteht darin, die Balance zwischen Delegation und Kontrolle herzustellen (vgl. z. B. Team FME 2013, S. 10). Eine gute Kommunikation auf persönlicher 


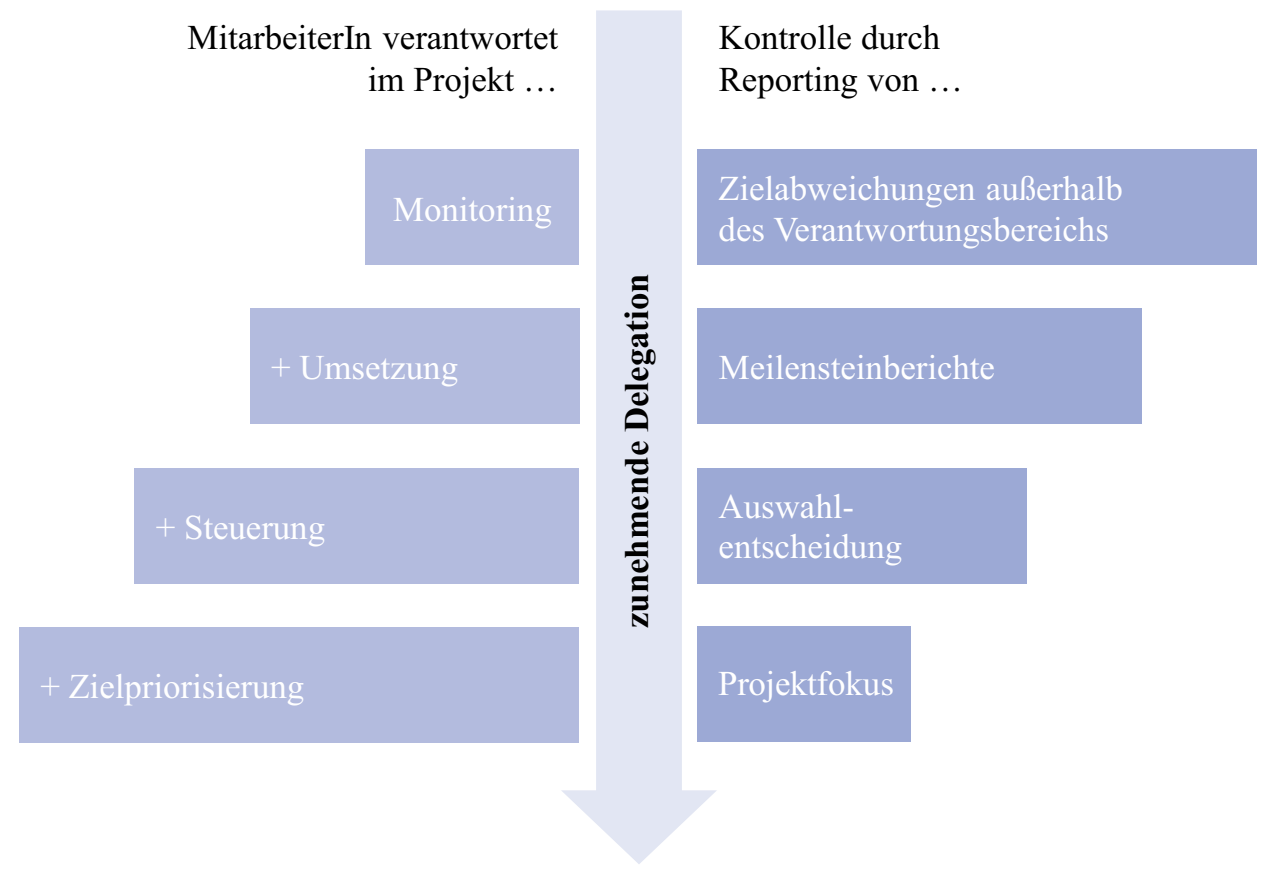

Abb. 3: Mögliche Stufen projekt-

bezogener Delegation

Ebene spielt dabei eine zentrale Rolle. So bietet es sich etwa an, regelmäßig Feedbackgespräche zu führen. Darüber hinaus muss es selbstverständlich sein, dass man seinen MitarbeiterInnen bei außergewöhnlichen Problemen stets für Hilfe zur Verfügung steht.

\section{Herausforderungen in der Praxis}

Wie jede organisatorische Veränderung stellt die Umverteilung von Tätigkeiten im Rahmen einer systematischen Delegation eine große Herausforderung dar. Diese Aufgabe ist umso anspruchsvoller, je länger sich die MitarbeiterInnen an bestimmte Arbeitsinhalte und -abläufe gewöhnen konnten. Versucht man, entsprechende Routinen zu durchbrechen, ist mit durchaus größeren Widerständen von Beteiligten zu rechnen. In letzter Konsequenz kann eine Änderung der Teamzusammensetzung notwendig werden. Aber selbst ohne solche Widrigkeiten fällt es mancher Führungskraft aus eigenem Antrieb schwer, das überschaubare Terrain operativer Tätigkeiten zu verlassen und sich ambiguitätsbehafteten, strategischen Aufgaben zuzuwenden (vgl. z. B. Stahl, 2003, S. 17 ff.).

Vor diesem Hintergrund kann ein externes Coaching ganz im Sinne eines die Effektivität und Effizienz fördernden Controllings (vgl. Ahn, 2003, S. 102) - dazu beitragen, das Delegationsproblem zu bewältigen. Seine Aufgaben bestehen

- in der Motivation der Führungskraft zur Änderung des Status Quo,

- in der Unterstützung der Umsetzung hier vorgestellter und weiterer Instrumente,

- in der Übernahme der Funktion als Blitzableiter für auftretende Konflikte und
- in der Sicherstellung des langfristigen Erfolgs der angestoßenen Veränderungsprozesse.

Mit Blick auf den letztgenannten Aspekt gilt es, die vorgenommene Tätigkeitsklassifikation von Zeit zu Zeit auf den Prüfstand zu stellen. Insbesondere ist zu verfolgen, inwieweit es gelingt, die Mitarbeiterpotenziale schrittweise auszubauen, um auf dieser Grundlage den Umfang und das Anspruchsniveau delegierter Aufgaben zu steigern.

\section{Literatur}

Ahn, H., Effektivitäts- und Effizienzsicherung - Controlling-Konzept und Balanced Scorecard, Frankfurt a. M. 2003.

Boneberg, I., Delegation, in: T. M. Steiger, E. Lippmann (Hrsg.), Handbuch Angewandte Psychologie für Führungskräfte, Berlin et al. 2008, S. 160171.

Convey, S. R., Die 7 Wege zur Effektivität - Prinzipien für persönlichen und beruflichen Erfolg, 39. Aufl., Offenbach 2016.

Gallo, A., Why aren't you delegating?, in: Harvard Business Review, 2012, Online im Internet: URL: https://hbr.org/2012/07/why-arent-you-deleg ating (Abrufdatum: 24.10.2017).

Knoblauch, J./Wöltje, H./Hausner, M. B./Kimmich, M./Lachmann, S., Zeitmanagement, 3. Aufl., Freiburg 2015.

Laux, H., Grundfragen der Organisation - Delegation, Anreiz und Kontrolle, Berlin/Heidelberg 1979.

Seiwert, L., Noch mehr Zeit für das Wesentliche - Zeitmanagement neu entdecken, 7. Aufl., München 2017.

Stahl, H. K., Unternehmer und Manager - Wie aktuell sind die beiden Rollen?, in: H. K. Stahl, H. H. Hinterhuber (Hrsg.), Erfolgreich im Schatten der Großen - Wettbewerbsvorteile für kleine und mittlere Unternehmen, Berlin 2003, S. 3-29.

Team FME, Successful delegation, Online im Internet: URL: https://www.g oogle.de/search?q=Team+FME\%2C+Successful+delegation\&ie=utf-8\&oe= utf-8\&client=firefox-b\&gfe_rd=cr\&dcr=0\&ei=207uWdTCBY3Z8AefwoKwCQ (Abrufdatum: 24.10.2017). 